\title{
Gauss-Hermite quadratures and accuracy of lattice Boltzmann models for non-equilibrium gas flows
}

\author{
Jianping Meng* and Yonghao Zhang ${ }^{\dagger}$ \\ Department of Mechanical Engineering, University of Strathclyde, Glasgow, G1 1 XJ, UK
}

(Dated: March 14, 2011)

\begin{abstract}
Recently, the kinetic theory based lattice Boltzmann (LB) models have been developed to model non-equilibrium gas flows. Depending on the order of quadratures, a hierarchy of LB models can be constructed which we have previously shown to be able to capture rarefaction effects in the standing shear wave problems. Here, we further examine the capability of high-order LB models in modeling non-equilibrium flows considering gas/surface interactions and their effect on the bulk flow. The Maxwellian gas/surface interaction model, which has been commonly used in other kinetic methods including direct simulation of Monte Carlo method, is used in the LB simulations. In general, the LB models with high-order Gauss-Hermite quadratures can capture flow characteristics in the Knudsen layer and higher-order quadratures give more accurate prediction. However, for the Gauss-Hermite quadratures, the present simulation results show that the LB models with the quadratures obtained from the even-order Hermite polynomials perform significantly better than those from the odd-order polynomials. This may be attributed to the zero-velocity component in the odd-order discrete set, which does not participate wall/gas collisions, and thus under-estimate wall effect.
\end{abstract}

PACS numbers: 05.10.-a, 47.45.-n, 47.61.-k

\section{INTRODUCTION}

Non-equilibrium gas flows are traditionally associated with spacecraft re-entry into planetary atmosphere and vacuum technologies. Recently, research in nonequilibrium gas dynamics is reignited because of rapid development of micro/nano-technologies and modern material processing techniques including laser fabrication processing and plasma etching [1-3]. In addition to traditional high-speed low-density non-equilibrium flows, the research interest in rarefied gas dynamics is extended to low-speed flows under standard ambient temperature and pressure. For all these non-equilibrium flows, the linear constitutive relation for stress, which is assumed in the Navier-Stokes equation, is no longer valid. The level of non-equilibrium in gas local flowfield can be classified by the following Knudsen number:

$$
K n=\frac{\lambda}{l} \approx \frac{\lambda}{Q} \frac{d Q}{d l},
$$

where $\lambda$ is the mean free path of the gas molecules, $l$ is the characteristic length scale of the flow system and $Q$ is a quantity of interest, such as the gas density, pressure or temperature. According to the Knudsen number, gas flow regimes can be divided into : the hydrodynamic regime $(K n<0.001)$, the slip regime $(0.001<K n<$ $0.1)$, the transition regime $(0.1<K n<10)$, and the free molecular flow regime $(K n>10)$. The Navier-Stokes equation is only valid in the hydrodynamic regime. Micro/nano devices often operate in the hydrodynamic, slip

\footnotetext{
* Email: jianping.meng@strath.ac.uk

$\dagger$ Author to whom correspondence should be addressed.

Email: yonghao.zhang@strath.ac.uk
}

and transition flow regimes with a range of Knudsen numbers in different parts of the device. Therefore, we need a computationally efficient method to solve the Boltzmann equation, which is valid in all the flow regimes.

For traditional high-speed applications, the direct simulation Monte Carlo (DSMC) method can provide accurate solutions for non-equilibrium gas flows. However, for low-speed flows with small Knudsen number, the DSMC method needs large statistical samples to reduce variance. Meanwhile, the direct solution of the Boltzmann equation is very complex due to the collisional integral.

The lattice Boltzmann (LB) framework has the potential to be an alternative method to simulate nonequilibrium gas flows. It was originally developed for hydrodynamics and was proved to be a viable numerical tool [4-7]. The LB models have been applied to a variety of important areas e.g., turbulence(e.g., Ref.[10]), multiphase flow, microfluidics and particle suspensions (see Ref.[8]). To construct appropriate LB models, there are different theoretical frameworks, e.g., entropic LB models $[9,11-13]$ and the Hermite expansion approach [21, 28-31]. Recently, non-equilibrium gas flows have become an active area of research for the LB method (e.g., Refs.[8, 14-21, 23-26, 42, 43]). Theoretically, it was shown that the LB model with discrete velocity set derived from high-order Gauss Hermite quadratures can provide a computationally efficient way of solving the Boltzmann model equation [21]. Higher-order LB models can asymptotically recover the Bhatnagar-Gross-Krook (BGK) equation[21].

The capability of high-order LB models for nonequilibrium gas has been reported. Analytical solutions for up to the D2Q16 model have been obtained for Couette and Poiseuille flows with finite Knudsen numbers $[23,25,42,43]$. Numerical experiments have also been conducted to further assess higher-order models up to the 
D2Q36 model [24]. It was concluded that higher-order LB methods can improve the capability in comparison with the standard LB method but the accuracy does not monotonically increase with the order of the GaussHermite quadrature. Specifically, the D2Q25 model performs poorer than the D2Q16 model[24]. For a standing shear wave problem where the gas/surface interactions are irrelevant, surprisingly, we found that the D2Q25 model performs better than the D2Q16 model, different from the observation made in microchannel flows [27]. As the LB model equation with a first order Hermite expansion for the equilibrium distribution function was shown to be sufficient to capture non-equilibrium effects at low Mach number both numerically [24] and theoretically [27], this contradictory observation of the high-order LB model capability may be caused by the quadrature effect in dealing with wall boundary. Therefore, in this work, we will further examine how various orders of Gauss-Hermite quadratures influence the treatment of essential physics of gas molecule/wall surface interactions.

\section{HIGH-ORDER LB MODELS}

High-order LB models for rarefied gas can be constructed by utilizing the Gauss-Hermite quadrature [21, $28-31]$. It is computationally very expensive to solve the full Boltzmann equation while model Boltzmann equations such as the BGK equation can produce sufficiently accurate results for macroscopic properties such as velocity, density, pressure, and stress. So LB models are often based on the BGK equation. The original BGK equation is:

$$
\frac{\partial f}{\partial t}+\boldsymbol{\xi} \cdot \nabla f+\boldsymbol{g} \cdot \nabla_{\xi} f=-\frac{p}{\mu}\left(f-f^{e q}\right),
$$

where $f$ denotes the distribution function, $\boldsymbol{\xi}$ the phase velocity, $p$ the pressure, $\boldsymbol{g}$ the body force and $\mu$ the gas viscosity. Using the well-known Chapman-Enskog expansion, the collision frequency can be represented by the ratio of pressure and gas viscosity, which is convenient to obtain the Knudsen number definition consistent with that of the hydrodynamic models. Without losing generality, we define the following non-dimensional variables:

$$
\begin{gathered}
\hat{\boldsymbol{r}}=\frac{\boldsymbol{r}}{l}, \hat{\boldsymbol{u}}=\frac{\boldsymbol{u}}{\sqrt{R T_{0}}}, \hat{t}=\frac{\sqrt{R T_{0}} t}{l}, \\
\hat{\boldsymbol{g}}=\frac{l \boldsymbol{g}}{R T_{0}}, \hat{\boldsymbol{\xi}}=\frac{\boldsymbol{\xi}}{\sqrt{R T_{0}}}, \hat{T}=\frac{T}{T_{0}},
\end{gathered}
$$

where $\boldsymbol{u}$ is the macroscopic velocity, $R$ the gas constant, $T$ the gas temperature, $T_{0}$ the reference temperature, $\boldsymbol{r}$ the spatial position and $l$ the characteristic length of the flow system. The symbol hat, which denotes dimensionless value, will hereinafter be omitted. The Knudsen number can be defined by using macroscopic properties as below:

$$
K n=\frac{\mu \sqrt{R T_{0}}}{p l}
$$

Based on these non-dimensional variables, the nondimensional form of the BGK equation becomes

$$
\frac{\partial f}{\partial t}+\boldsymbol{\xi} \cdot \nabla f+\boldsymbol{g} \cdot \nabla_{\xi} f=-\frac{1}{K n}\left(f-f^{e q}\right),
$$

where the Maxwell distribution in $D$-dimensional Cartesian coordinates can be written as

$$
f^{e q}=\frac{\rho}{(2 \pi T)^{D / 2}} \exp \left[\frac{-(\boldsymbol{\xi}-\boldsymbol{u})^{2}}{2 T}\right] .
$$

For solving Eq.(5), the velocity space can be firstly discretized by projecting the distribution function onto a functional space spanned by the orthogonal Hermite basis[32]:

$$
f(\boldsymbol{r}, \boldsymbol{\xi}, t) \approx f^{N}(\boldsymbol{r}, \boldsymbol{\xi}, t)=\omega(\boldsymbol{\xi}) \sum_{n=0}^{N} \frac{1}{n !} \boldsymbol{a}^{(n)}(\boldsymbol{r}, t) \boldsymbol{\chi}^{(n)}(\boldsymbol{\xi})
$$

where $\chi^{(n)}$ is the $n$th order Hermite polynomial, and $\omega(\boldsymbol{\xi})$ is the weight function, which are given by

$$
\begin{gathered}
\chi^{(n)}(\boldsymbol{\xi})=\frac{(-1)^{n}}{\omega(\boldsymbol{\xi})} \nabla^{n} \omega(\boldsymbol{\xi}), \\
\omega(\boldsymbol{\xi})=\frac{1}{(2 \pi)^{D / 2}} \mathrm{e}^{-\xi^{2} / 2} .
\end{gathered}
$$

The coefficients $\boldsymbol{a}^{(n)}$ are

$$
\begin{aligned}
\boldsymbol{a}^{(n)} & =\int f \boldsymbol{\chi}^{(n)} d \boldsymbol{\xi} \approx \int f^{(N)} \boldsymbol{\chi}^{(n)} d \boldsymbol{\xi} \\
& =\sum_{\alpha=1}^{d} \frac{w_{\alpha}}{\omega\left(\boldsymbol{\xi}_{\alpha}\right)} f^{(N)}\left(\boldsymbol{r}, \boldsymbol{\xi}_{\alpha}, t\right) \boldsymbol{\chi}^{(n)}\left(\boldsymbol{\xi}_{\alpha}\right) .
\end{aligned}
$$

The equilibrium distribution should also be expanded as[21]

$$
f^{e q} \approx \omega(\boldsymbol{\xi}) \sum_{n=0}^{N} \frac{1}{n !} \boldsymbol{a}_{e q}^{(n)} \chi^{(n)}(\boldsymbol{\xi})
$$

where the coefficient $a_{e q}^{(n)}$ for the equilibrium distribution is

$$
\boldsymbol{a}_{e q}^{(n)}=\int f^{e q} \boldsymbol{\chi}^{(n)} d \boldsymbol{\xi}
$$

$w_{\alpha}$ and $\boldsymbol{\xi}_{\alpha}, a=1, \cdots, d$, are the weights and abscissae of a Gauss-Hermite quadrature of degree $\geq 2 N$ respectively. Therefore, the Maxwell distribution is approximated by up to $N$ Hermite polynomials. The body force term $F(\boldsymbol{r}, \boldsymbol{\xi}, t)=\boldsymbol{g} \cdot \nabla_{\xi} f$ can also be approximated as[21]

$$
F(\boldsymbol{r}, \boldsymbol{\xi}, t)=\omega \sum_{n=1}^{N} \frac{1}{(n-1) !} \boldsymbol{g} \boldsymbol{a}^{(n-1)} \boldsymbol{\chi}^{(n)}
$$


It was shown that the first-order expansion on the equilibrium distribution is sufficient for LB model to capture isothermal and incompressible non-equilibrium flow phenomena[27]. Therefore, as low speed flows are mainly considered in this work, the standard form of second order approximation of the equilibrium distribution and the body force will be used, as given below:

$f^{e q} \approx \omega(\boldsymbol{\xi}) \rho\left\{1+\boldsymbol{\xi} \cdot \boldsymbol{u}+\frac{1}{2}\left[(\boldsymbol{\xi} \cdot \boldsymbol{u})^{2}-u^{2}+(T-1)\left(\xi^{2}-D\right)\right]\right\}$,

$$
F(\boldsymbol{r}, \boldsymbol{\xi}, t) \approx \omega(\boldsymbol{\xi}) \rho\{\boldsymbol{g} \cdot \boldsymbol{\xi}+(\boldsymbol{g} \cdot \boldsymbol{\xi})(\boldsymbol{u} \cdot \boldsymbol{\xi})-\boldsymbol{g} \cdot \boldsymbol{u}\},
$$

where $T$ should be unity for isothermal problems and $\rho$ is constant for incompressible problems.

Based on the Gauss-Hermite quadratures, various order discrete velocity sets can be constructed [21, 22], which are the upmost important factor in determining model accuracy [27]. Once the discrete velocity set is chosen, Eq.(5) can be discretized as

$$
\frac{\partial f_{\alpha}}{\partial t}+\boldsymbol{\xi}_{\alpha} \cdot \nabla f_{\alpha}=-\frac{1}{K n}\left(f_{\alpha}-f_{\alpha}^{e q}\right)+g_{\alpha}
$$

where

$$
\begin{gathered}
f_{\alpha}=w_{\alpha} f\left(\boldsymbol{r}, \boldsymbol{\xi}_{\alpha}, t\right) / \omega\left(\boldsymbol{\xi}_{\alpha}\right), \\
f_{\alpha}^{e q}=w_{\alpha} f^{e q}\left(\boldsymbol{r}, \boldsymbol{\xi}_{\alpha}, t\right) / \omega\left(\boldsymbol{\xi}_{\alpha}\right),
\end{gathered}
$$

and

$$
g_{\alpha}=w_{\alpha} F\left(\boldsymbol{r}, \boldsymbol{\xi}_{\alpha}, t\right) / \omega\left(\boldsymbol{\xi}_{\alpha}\right) .
$$

Therefore, the LB equation, i.e. Eq.(16), is now obtained by discretizing Eq.(5) in the velocity space. In one-dimensional case, one can obtain $\xi_{\alpha}$ by solving the roots of one-dimensional Hermite polynomials and the corresponding weights are determined by:

$$
w_{\alpha}=\frac{n !}{\left[n \chi^{n-1}\left(\xi_{\alpha}\right)\right]^{2}} .
$$

For higher dimensions, the quadrature can be constructed by using the "production" formulae [21]. In this paper, we will assess the LB models in 2-dimension with the Gauss-Hermite quadratures corresponding to $9, \quad 16,25,36,49$ and 64 discrete velocities. We name these models as D2Q9, D2Q16, D2Q25, D2Q36, D2Q49, and D2Q64 according to the conventional LB terminology[46]. For convenience, the accuracy of a quadrature will be represented by its Hermite polynomial order $n$ hereinafter while its exact algebraic accuracy is $2 n-1$. For instance, the Gauss-Hermite quadrature of D2Q9 model is from the $3^{r d}$-order Hermite polynomial, we call it $3^{r d}$-order accuracy while its algebraic accuracy is the $5^{\text {th }}$-order. Similarly, D2Q16 is $4^{\text {th }}$-order, D2Q25, $5^{\text {th }}$-order, D2Q36, $6^{t h}$-order, D2Q49, $7^{t h}$-order and D2Q64 $8^{\text {th }}$-order.

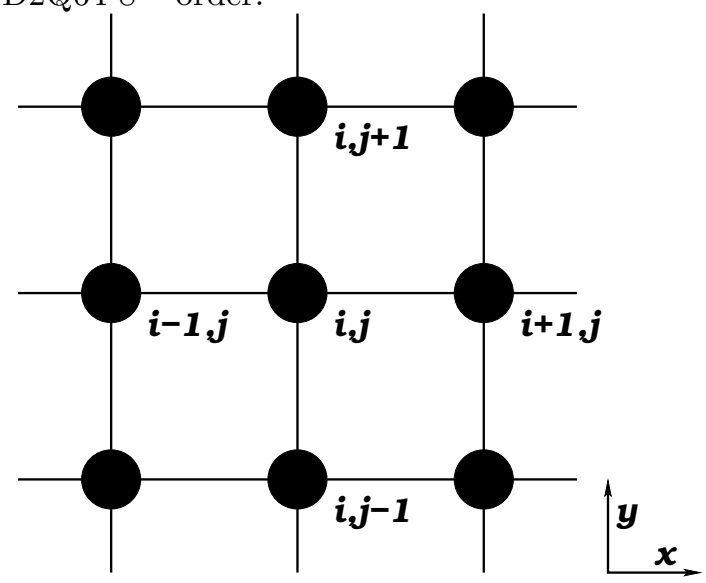

FIG. 1. Schematic diagram of square lattices.

\section{NUMERICAL IMPLEMENTATION AND BOUNDARY CONDITION}

\section{A. Numerical implementation}

Various numerical schemes can be used to solve Eq.(16). For some high-order LB models with the noninteger-valued discrete sets, one has to choose a numerical scheme to break the tie between the time step and the lattice spacing used in the standard LB simulation. So they are essentially off-lattice models. As some discontinuities may occur at wall surface in the following simulations, we will employ the forward Euler time-marching method and the 2nd TVD scheme for space discretization (see Fig.1) for Eq.(16)[33-35]. According to the characteristics of problems, one can also choose any other appropriate numerical method to solve Eq.(16).

Let $f_{\alpha, i}^{n, j}$ denote the distribution function value $f_{\alpha}$ at the nth time step in the node $x_{i}, y_{j}$ (see Fig.1), the scheme can be written as

$$
f_{\alpha, i}^{n+1, j}=f_{\alpha, i}^{n, j}-\frac{\xi_{\alpha x} \delta_{t}}{\delta_{x}}\left[\mathcal{F}_{\alpha, i+1 / 2}^{n, j}-\mathcal{F}_{\alpha, i-1 / 2}^{n, j}\right]-\frac{\xi_{\alpha y} \delta_{t}}{\delta_{y}}\left[\mathcal{F}_{\alpha, i}^{n, j+1 / 2}-\mathcal{F}_{\alpha, i}^{n, j-1 / 2}\right]+\frac{\delta_{t}}{K n}\left(f_{\alpha, i}^{e q, n, j}-f_{\alpha, i}^{n, j}\right)+g_{\alpha} \delta_{t},
$$

where $\delta_{x}$ and $\delta_{y}$ are the uniform grid spacing, and and $\delta_{t}$ is the time step, $\xi_{\alpha x}$ and $\xi_{\alpha y}$ denote the phase velocity 
component at the $x$ and $y$ coordinates. The outgoing and incoming fluxes in the node $(i, j)$ (see Fig.1) are

$$
\begin{gathered}
\mathcal{F}_{\alpha, i+1 / 2}^{n, j}=f_{\alpha, i}^{n, j}+\frac{1}{2}\left(1-\frac{\xi_{\alpha x} \delta_{t}}{\delta_{x}}\right)\left[f_{\alpha, i+1}^{n, j}-f_{\alpha, i}^{n, j}\right] \Psi\left(\Theta_{\alpha, i}^{n}\right) \\
\mathcal{F}_{\alpha, i-1 / 2}^{n, j}=\mathcal{F}_{\alpha,(i-1)+1 / 2}^{n, j}, \\
\mathcal{F}_{\alpha, i}^{n, j+1 / 2}=f_{\alpha, i}^{n, j}+\frac{1}{2}\left(1-\frac{\xi_{\alpha y} \delta_{t}}{\delta_{y}}\right)\left[f_{\alpha, i}^{n, j+1}-f_{\alpha, i}^{n, j}\right] \Psi\left(\Theta_{\alpha}^{n, j}\right), \\
\mathcal{F}_{\alpha, i}^{n, j-1 / 2}=\mathcal{F}_{\alpha, i}^{n,(j-1)+1 / 2},
\end{gathered}
$$

where

$$
\begin{gathered}
\Theta_{\alpha, i}^{n}=\frac{f_{\alpha, i}^{n, j}-f_{\alpha, i-1}^{n, j}}{f_{\alpha, i+1}^{n, j}-f_{\alpha, i}^{n, j},} \\
\Theta_{\alpha}^{n, j}=\frac{f_{\alpha, i}^{n, j}-f_{\alpha, i}^{n, j-1}}{f_{\alpha, i}^{n, j+1}-f_{\alpha, i}^{n, j}},
\end{gathered}
$$

and the minmod flux limiter is

$$
\Psi(\Theta)=\max [0, \min (1, \Theta)]
$$

\section{B. Diffuse reflection boundary condition}

In the flowfield adjacent to the surface, gas molecules have more chance to collide with the wall surface, so that an appropriate gas/surface interaction model is important. Traditionally, the simple diffuse reflection model, developed by Maxwell in 1879 [36], which has been proven to be sufficiently accurate for simulating nonequilibrium flows with a broad range of Knudsen numbers. Therefore, the Maxwellian diffuse reflection model will be used here and its implementation in LB models has been reported in Ref.[37]. The numerical implementation of the Maxwellian diffuse reflection model in LB simulation has also been discussed in Refs. [33, 38]. In this work, the Version 1 of boundary conditions in Ref.[33] will be employed.

For convenience, we assume

$S \approx w_{\alpha}\left\{1+\boldsymbol{\xi}_{\alpha} \cdot \boldsymbol{u}+\frac{1}{2}\left[\left(\boldsymbol{\xi}_{\alpha} \cdot \boldsymbol{u}\right)^{2}-u^{2}+(T-1)\left(\xi_{\alpha}^{2}-D\right)\right]\right\}$,

so that $f_{\alpha}^{e q}$ is equal to $\rho S$. As we conduct the discretization along a Cartesian coordinate system (see Fig.2), the treatment of boundary can be described as

$$
f_{\alpha, k}^{0}=\rho_{W, k} S\left(T_{W, k}, \boldsymbol{u}_{W, k}\right) \quad \boldsymbol{\xi}_{\alpha} \cdot \boldsymbol{n}>0,
$$

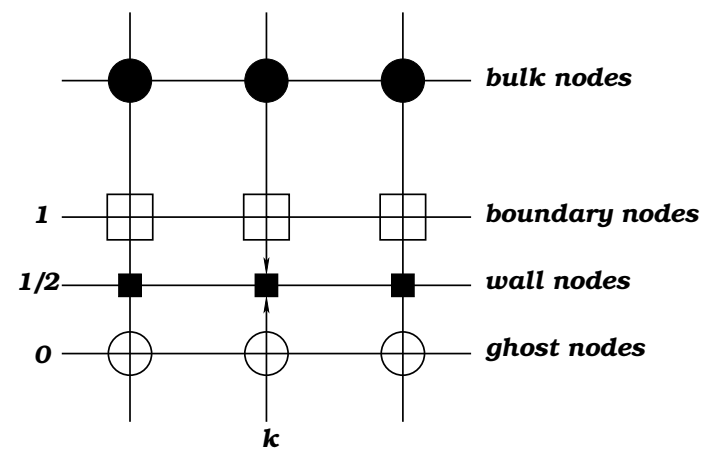

FIG. 2. Schematic illustration of wall boundary treatment.

$$
\rho_{W, k}=\frac{\sum_{\left(\boldsymbol{\xi}_{\alpha} \cdot \boldsymbol{n}\right)<0}\left|\boldsymbol{\xi}_{\alpha} \cdot \boldsymbol{n}\right| f_{\alpha, k}^{1}}{\sum_{\left(\boldsymbol{\xi}_{\alpha} \cdot \boldsymbol{n}\right)>0}\left|\boldsymbol{\xi}_{\alpha} \cdot \boldsymbol{n}\right| S\left(T_{W, k}, \boldsymbol{u}_{W, k}\right)},
$$

where the subscript $W$ denotes the computational nodes at the wall, $\rho_{W, k}$ denotes the density on the wall nodes $k$ (see Fig.2), $T_{W, k}$ the temperature, $\boldsymbol{u}_{W, k}$, the velocity, $\boldsymbol{n}$, the unity normal vector to the wall. In this version of boundary condition, the distribution functions in the ghost nodes are assumed to be identical to those on the corresponding wall nodes.

\section{NUMERICAL RESULTS AND DISCUSSION}

Significant efforts have been devoted to develop or examine the capability of LB models for finite Knudsen number flows e.g., [8, 14-21, 23-26, 42, 43]. Some works concentrated on proposing appropriate gas molecule/wall interaction models to capture the slip velocity, such as the bounce-back and specular reflection schemes or a combination of them [17, 39-41]. Further attention was attracted on modifications to relaxation dynamics $[19,20$, $42,44]$. An interesting modification is the so-called "effective relaxation time" approach where the relaxation time is correlated to the effective mean free path which 
takes account of the effect of gas molecule/surface collisions. It can successfully extend the capability of the standard LB models for simulating non-equilibrium flows with a broad range of Knudsen numbers [19, 20]. Ref.[21] has revealed that LB models can be derived systematically from the BGK equation, so that LB models can also be considered as an approximation of the BGK equation which is beyond the Navier Stokes hydrodynamics. Indeed the model equation of LB method is essentially a truncated BGK equation. With the first-order expansion on the equilibrium distribution function, it is the same as the linearized BGK equation. Therefore, Eq.(5) with the first order Hermite expansion is already sufficiently accurate for a broad range of rarefied problems[27]. Higherorder expansion terms should be mainly responsible for finite Mach number effects which turn out to be another important issue for LB models. Meanwhile, the discrete velocity set is of great importance for capturing non-equilibrium effects. For a standing shear wave problem where the wall effect does not exist, we have shown that the high-order LB models with moderate order quadratures (e.g., D2Q16) can provide good results. While the gas molecule/surface interactions become important, the situation becomes more complicated because gas molecular/wall collisions play a dominant role. Because of the Knudsen layer which is the flowfield adjacent to the wall surface, the momentum and energy cannot be transferred effectively from the wall to the bulk flow and vice versa. The discontinuity of macroscopic quantities will occur, i.e. the velocity slip and temperature jump, which will make non-equilibrium gas flow simulation a challenging task. Although Kim et al.([24]) have examined high-order LB models for Couette and Poiseuille flows, we will further clarify the relation between GaussHermite quadratures and LB model accuracy in capturing non-equilibrium effects in rarefied flows especially in the Knudsen layer.

\section{A. Kramer's problem}

The classic Kramers' problem is often used to assess model capability in capturing the flow characteristics in the Knudsen layer. This problem consists of finding the molecular distribution function of a gas which fills the half-space $(y>0)$ bounded by a plate at $y=0$ (see Fig.3). A constant shear rate along the plate is applied at $y \rightarrow \infty$. In the simulations, the wall at $y=0$ is fixed and a constant shear rate is applied at $y=200 \lambda$. The Maxwellian diffuse reflection boundary condition is employed for the fixed wall.

Fig. 4 compares the LB simulation results with the data given by Loyalka et al. [45]. Similar to the standing-shear wave problem [27], discrete velocity sets are proved to be of importance to accurately capture non-equilibrium effects in the Knudsen layer. When a gas becomes sufficiently rarefied, the collisions of high speed gas molecules with the wall surface will be mainly responsible for mo-

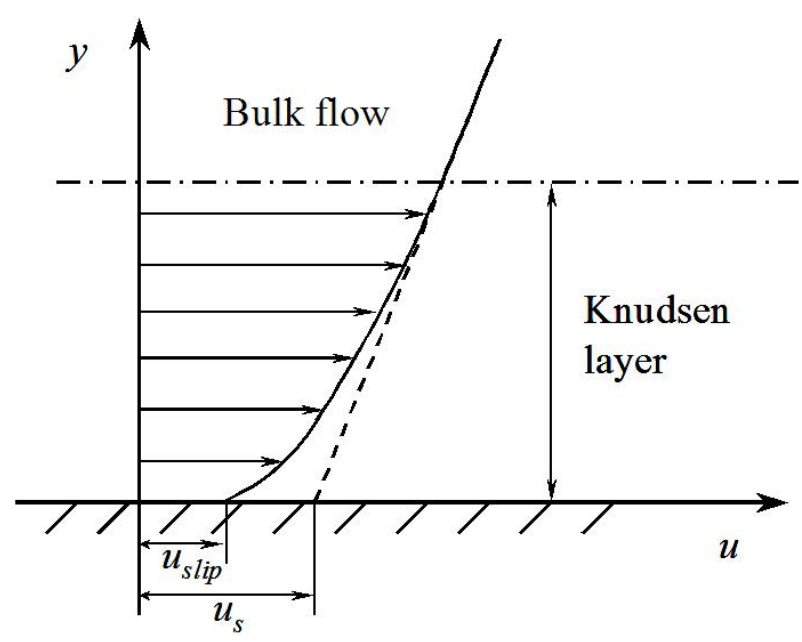

FIG. 3. Schematic diagram showing the microscopic slip $\left(u_{\text {slip }}\right)$ and macroscopic slip $\left(u_{s}\right)$ for the Kramers' problem.
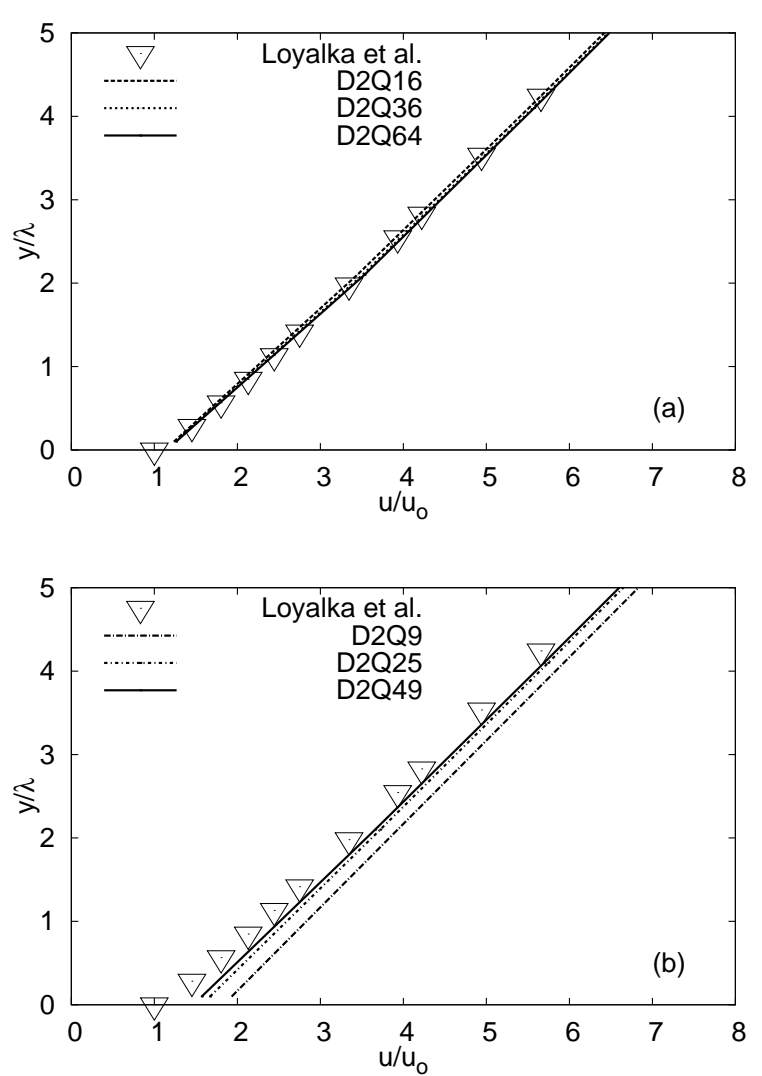

FIG. 4. The velocity profile of Kramers' problem with the diffuse reflection boundary condition. The symbols are the data from Loyalka et al.[45]. Here, the velocity is normalized by the reference velocity $u_{o}=-\sigma_{x y} \lambda / \mu$. The space quantity is normalized by the mean free path. The data in Ref.[45] were presented with the mean free path difined by $\mu \sqrt{2 R T} / p$. They are converted to be consistent with the present definition. 


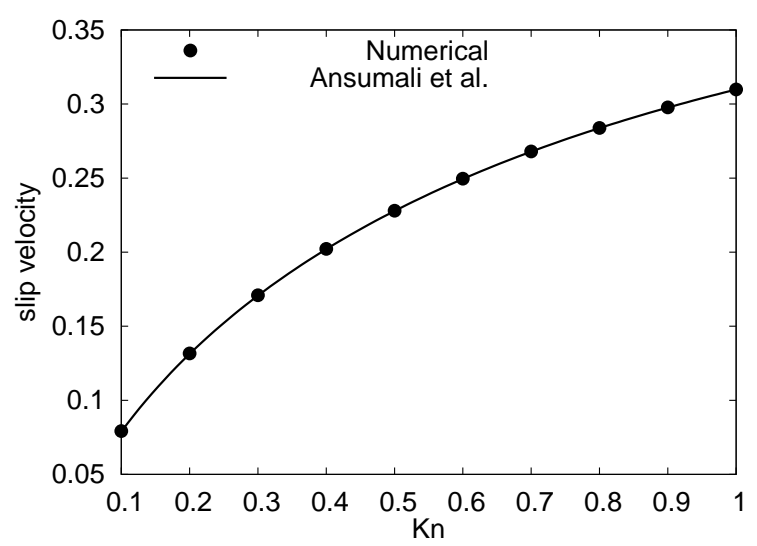

FIG. 5. The slip velocities of planar Couette flow, comparing our numerical simulation with the analytic solution given by Ansumali et al. [23], where both numerical and analytical solutions are based on the D2Q16 model.

mentum and energy transfer as the molecules with low speed molecules will less frequently collide with the wall surface. Therefore, higher order discrete velocity sets can help to improve model accuracy since the effects of high speed gas molecules can be better represented.

It is also interesting to note that the LB models with even-order Gauss-Hermite quadratures (D2Q16, D2Q36 and D2Q64) provide results in excellent agreement with the data reported in the Ref. [45]. By contrast, the LB models with odd-order quadratures, i.e. D2Q9, D2Q25, D2Q49 models, perform significantly worse than those with even-order quadratures despite the higher-order models still provide better results. This interesting observation, i.e. the discrete velocity sets from the even order Hermite polynomials show much better performance than those from odd order ones, may attribute to the fact that the odd-order quadratures contain a zero discrete velocity. A physical explanation is that the zero discrete velocity component in the discrete velocity sets from odd polynomials, which represents a significant portion of gas molecules, do not contribute to momentum and energy transfer through collisions with the wall surface. Therefore, gas molecule/surface interactions are under-estimated which leads to over-prediction of slip velocity, see Fig.4. As a result, we may conclude that the discrete velocity set from odd polynomials do not appropriately represent gas molecular speeds. This observation will be further confirmed in the following simulations.

\section{B. Couette flow}

With a simple geometry configuration, the Couette flow represents many realistic shear dominant applications, e.g. hard-disc driver reader heads, micro turbines and gas bearings. The Maxwellian diffuse reflection boundary condition is again employed to describe gas/surface interactions. The upper and lower plates are
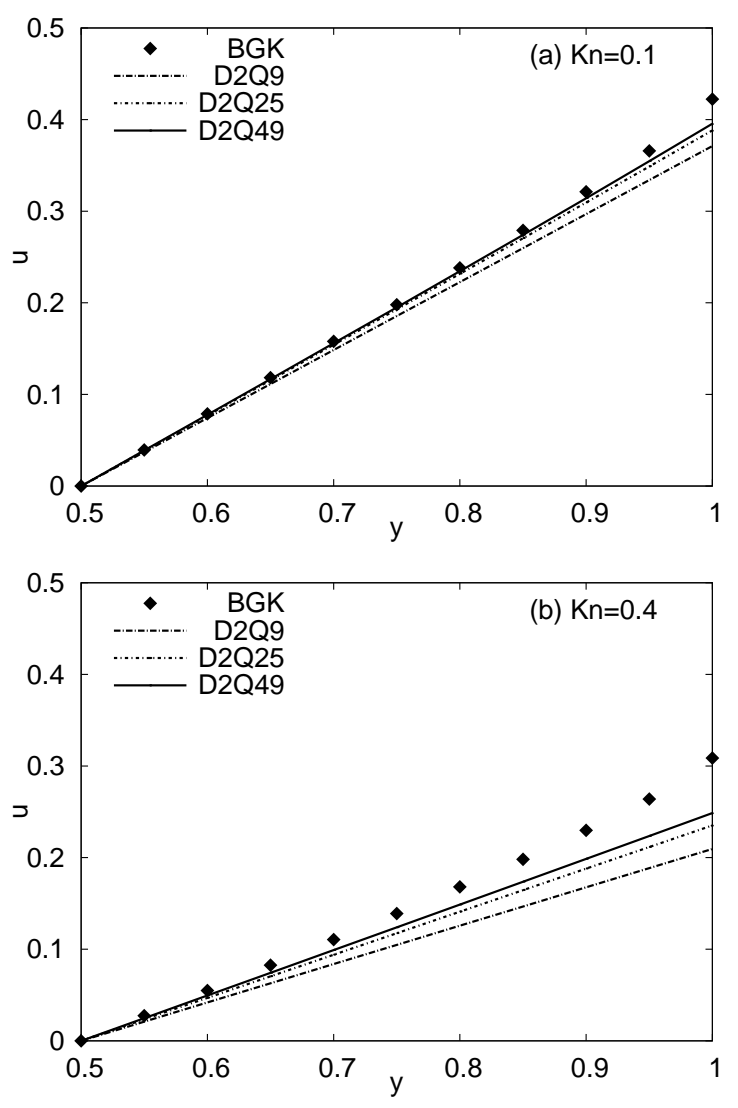

FIG. 6. Nondimensional velocity profiles for planar Couette flows where the velocity is normalized by the velocity difference between the two plates. Comparison of the odd-order LB models with the linearized BGK solution.

set to be moving oppositely with the same velocity magnitude.

In Fig. 5, the slip velocities from our numerical simulations are compared with the analytic solutions given by Ansumali et al.[23] for the D2Q16 model, where excellent agreement can be clearly observed. Similar to the Kramer's problem, higher-order LB models provide better simulation results when compared with the solution of the linearized BGK equation and the DSMC data (see, Figs. 6-7).

The D2Q16 model performs well for non-equilibrium flows with the Knudsen number up to 0.5 , while the $8^{\text {th }}$ order D2Q64 model give excellent results even at $\mathrm{Kn}=1.0$. The reason is that the higher speed gas molecules are better represented by higher-order discrete velocities. In particular, we can see that the curvature of velocity profile close to the wall region is captured well, which is a typical non-equilibrium phenomenon in the Knudsen layer. The Navier Stokes equation will predict a linear velocity profile across the two plates. Again, we find that the LB models with the discrete velocity sets from the odd order Hermite polynomials give worse results. Fig. 8 clearly show that the $4^{t h}$-order D2Q16 LB model is even significantly better than the $7^{\text {th }}$-order D2Q49 

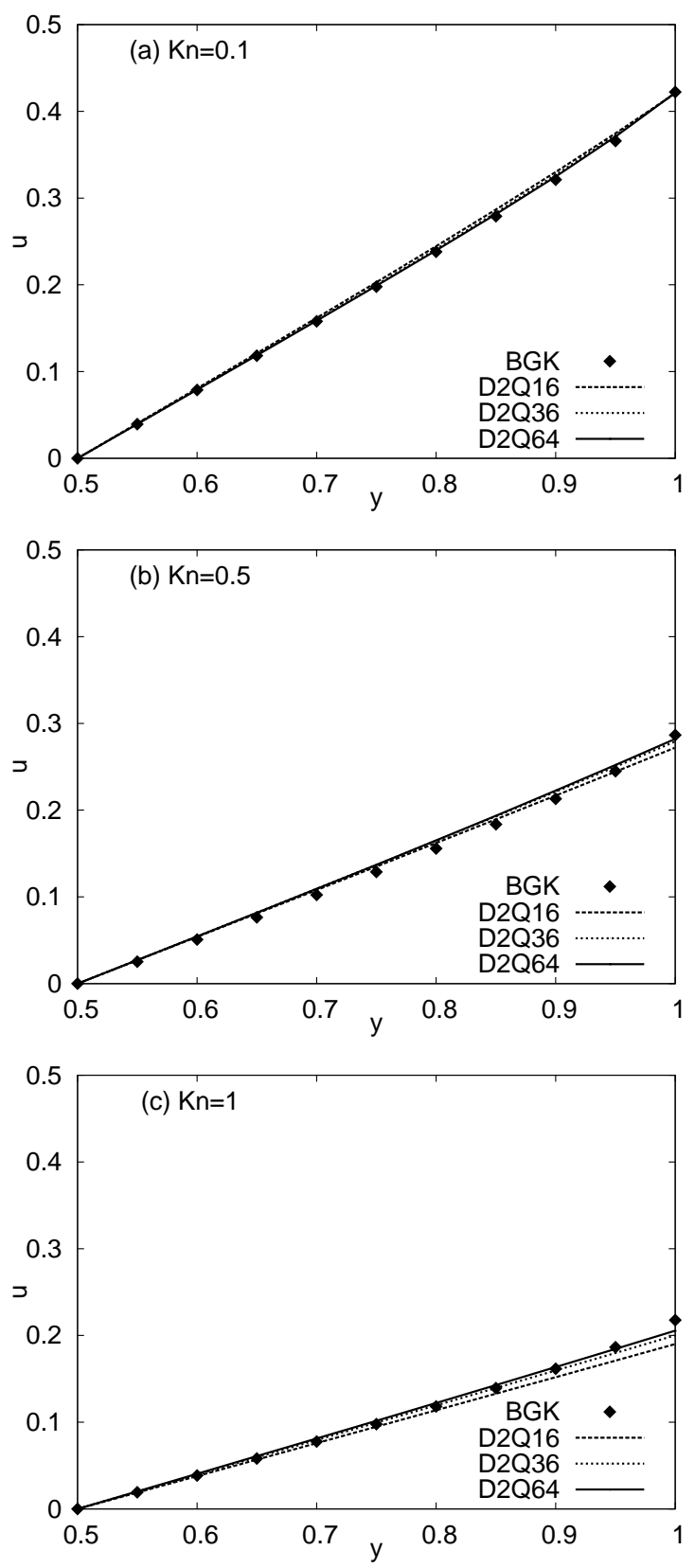

FIG. 7. Nondimensional velocity profiles for planar Couette flows where the velocity is normalized by the velocity difference between the two plates. Comparison of the even-order LB models with the linearized BGK solution.

model. Similarly, Kim et al. [24] also reported that the D2Q16 model can perform better than the D2Q25 model. When the Knudsen number increases via reducing density or the system characteristic length, high speed gas molecules will more frequently collide with the wall surface to effectively transfer momentum and energy between the flowfield and the wall. Such mechanisms are less reflected in the lower-order LB models and the LB models with odd-order quadratures (the zero discrete velocities do not participate the collisions). Therefore, these models over-estimate the slip velocity, i.e. the influence of wall is under estimated, see Figs. 7-8.

\section{Force-driven Poiseuille flow}

Finally, we will assess the models for fully-developed force-driven Poiseuille flows where the two parallel plates are located at $y=0$ and $y=l$. The applied body force in the streamwise direction is small, so that the flow can be considered as incompressible.

When compared with the linearized BGK and DSMC data, see Figs. 9-10, we can again confirm that the higherorder LB models predict non-equilibrium Poiseuille flows better while the even order models perform better than the odd-order ones. However, the presence of body force increases the complexity of the rarefaction effect. Fig. 9 shows that the D2Q64 model significantly over estimates the slip velocity at the wall at the Knudsen number as low as 0.8. Meanwhile, the odd-order models are even worse, which significantly departure from the direct solution of the linearized BGK equation at the Knudsen number as low as 0.3 , see Fig. 10. Fig. 11 confirms the importance of choosing even-order Gauss-Hermite quadratures for LB simulation of non-equilibrium gas flows.

Kim et al.[24] found that the D2Q9 and D2Q25 can qualitatively capture the Knudsen minimum while the even-order models such as D2Q16 and D2Q36 fail. We can confirm their observation as shown in Fig. 12 where even D2Q64 cannot predict the Knudsen minimum. However, we also notice that the even-order models, quantitatively, provide more accurate results. To test whether higher-order even-order models can capture the Knudsen minimum, we have tested extreme high-order quadratures: D2Q4761 and D2Q4624 (see Fig. 12). Note: we are not suggesting to use such high-order models; this test is just to show that the even-order model (D2Q4624) cannot only capture the Knudsen minimum but also achieve more accurate results than the similar-order oddorder one (D2Q4761); indeed, such high-order model will be computationally costly(the D2Q4761 model takes 748 times longer than the D2Q9 for one computing time step in this case), which is not ideal for practical computations. It indicates that, with increasing order, the even order models are actually approaching the correct solution quicker than the odd-order ones. The quantitatively better results of even orders (e.g., D2Q36, D2Q64) are due to better representation of gas molecular speeds, in comparison with the corresponding odd-order models.

\section{CONCLUDING REMARKS}

The LB models with high order Gauss-Hermite quadratures are able to capture non-equilibrium phenomena in gas flows. For the Guass-Hermite quadratures, the LB models with higher-order quadratures perform better while the even-order LB models may be preferred as the 
high-speed gas molecules are more appropriately represented by the discrete velocities which is important to momentum and energy transfer between the wall surface and the gas. However, as shown in Ref.[48], the discrete velocity sets obtained via a different route can perform quite differently, which indicates that more efforts are required to fully understand optimal quadratures for describing non-equilibrium effects.

\section{ACKNOWLEDGMENTS}

The authors would like to thank Jason Reese, Xiaojun Gu, Yuehong Qian and Nicolas Hadjiconstantinou for many informative discussions. The research leading to these results has received funding from the European Community's Seventh Framework Programme (ITN - FP7/2007-2013) under grant agreement no. 215504. This work is also financially supported by the Engineering and Physical Sciences Research Council U.K. under Grants No. EP/F028865/1.
$08(1)$

[1] C. M. Ho and Y. C. Tai, Annu. Rev. Fluid Mech. 30, 579 (1998).

[2] C. M. Ho and Y. C. Tai, J. Fluid Eng 118, 437 (1996).

[3] G. Karniadakis, A. Beskok, and N. Aluru, Microflows and Nanoflows: Fundamentals and Simulation (Interdisciplinary Applied Mathematics) (Springer, 2005).

[4] S. Succi, I. V. Karlin, and H. Chen, Rev. Mod. Phys. 74, 1203 (2002).

[5] S. Y. Chen and G. D. Doolen, Annu. Rev. Fluid Mech. 30, 329 (1998).

[6] Y. H. Qian, S. Succi, and S. Orszag, Annu. Rev. Comput. Phys. III, 192 (1995).

[7] R. Benzi, S. Succi, and M. Vergassola, Phys. Rep. 222 145 (1992).

[8] C. K. Aidun and J. R. Clausen, Annu. Rev. Fluid Mech. 42, 439 (2010).

[9] S. S. Chikatamarla and I. V. Karlin, Phys. Rev. Lett. 97, 190601 (2006).

[10] S. S. Chikatamarla, C. E. Frouzakis, I. V. Karlin, A. G. Tomboulides and K. B.Boulouchos, J. Fluid Mech. 656, 298(2010).

[11] S. Ansumali, I. V. Karlin, and H. C. Öttinger, Europhys. Lett. 63, 798 (2003).

[12] S. Ansumali and I. V. Karlin, Phys. Rev. E 62, 7999 (2000).

[13] I. V. Karlin, A. Ferrante, and H. C. Öttinger, Europhys. Lett. 47, 182 (1999).

[14] I. V. Karlin and S. Ansumali, Phys. Rev. E 76, 025701 (2007).

[15] Y. H. Zhang, R. S. Qin, and D. R. Emerson, Phys. Rev. E 71, 047702 (2005).

[16] F. Toschi and S. Succi, Europhys. Lett. 69, 549 (2005).

[17] M. Sbragaglia and S. Succi, Phys. Fluids 17, 093602(2005).

[18] M. Sbragaglia and S. Succi, Europhys. Lett. 73, 370 (2006).

[19] G. H. Tang, Y. H. Zhang, and D. R. Emerson, Phys. Rev. E 77, 046701 (2008).

[20] Y. H. Zhang, X. J. Gu, R. W. Barber, and D. R. Emerson, Phys. Rev. E 74, 046704 (2006).

[21] X. W. Shan, X. F. Yuan, and H. D. Chen, J. Fluid Mech. 550, 413 (2006)

[22] X. W. Shan, Phys. Rev. E 81, 036702 (2010).

[23] S. Ansumali, I. V. Karlin, S. Arcidiacono, A. Abbas, and N. I. Prasianakis, Phys. Rev. Lett. 98, 124502(2007).
[24] S. H. Kim, H. Pitsch, and I. D. Boyd, J. Comput. Phys. 227, 8655 (2008).

[25] W. P. Yudistiawan, S. Ansumali, and I. V. Karlin, Phys. Rev. E 78, 016705 (2008).

[26] Z. L Guo, T. S. Zhao, and Y. Shi, J. Appl. Phys. 99, 074903 (2006).

[27] J. P. Meng and Y. H. Zhang, J. Comput. Phys. 230, 835 (2011).

[28] X. Y. He and L. S. Luo, Phys. Rev. E 55, R6333 (1997).

[29] X. Y. He and L. S. Luo, Phys. Rev. E 56, 6811 (1997).

[30] X. W. Shan and X. Y. He, Phys. Rev. Lett. 80, 65 (1998).

[31] N. S. Martys, X. W. Shan, and H. D. Chen, Phys. Rev. E 58, 6855 (1998).

[32] H. Grad, Commun. Pure Appl. Math. 2, 331 (1949).

[33] V. Sofonea, J. Comput. Phys. 228, 6107 (2009).

[34] V. Sofonea, A. Lamura, G. Gonnella, and A. Cristea, Phys. Rev. E 70, 046702 (2004).

[35] E. F. Toro, Riemann solvers and numerical methods for fluid dynamics - A practical introduction (Springer, Berlin, 1999).

[36] J. C. Maxwell, Phil. Trans. R. Soc. Lond. 170, 231 (1879).

[37] S. Ansumali and I. V. Karlin, Phys. Rev. E 66, 026311 (2002).

[38] V. Sofonea and R. F. Sekerka, J. Comput. Phys. 207, 639 (2005)

[39] X. B. Nie, G. D. Doolen, and S. Y. Chen, J. Stat. Phys. 107, 279 (2002).

[40] S. Succi, Phys. Rev. Lett. 89, 064502 (2002).

[41] C. Y. Lim, C. Shu, X. D. Niu, and Y. T. Chew, Phys. Fluids 14, 2299 (2002).

[42] S. H. Kim, H. Pitsch, and I. D. Boyd, Phys. Rev. E 77, 026704(2008).

[43] S. H. Kim and H. Pitsch, Phys. Rev. E 78, 016702(2008).

[44] G. H. Tang, X. J. Gu, R. W. Barber, D. R. Emerson, and Y. H. Zhang, Phys. Rev. E 78, 026706 (2008).

[45] S. K. Loyalka, N. Petrellis, and T. S. Storvick, Phys. Fluids 18, 1094 (1975).

[46] Y. H. Qian, D. D'Humières, and P. Lallemand, Europhys. Lett 17, 479 (1992).

[47] K. Aoki, S. Takata and T. Nakanishi, Phys. Rev. E 65, 026315 (2002).

[48] W. P. Yudistiawan, S. K. Kwak, D. V. Patil, and S. Ansumali, Phys. Rev. E 82, 046701 (2010). 

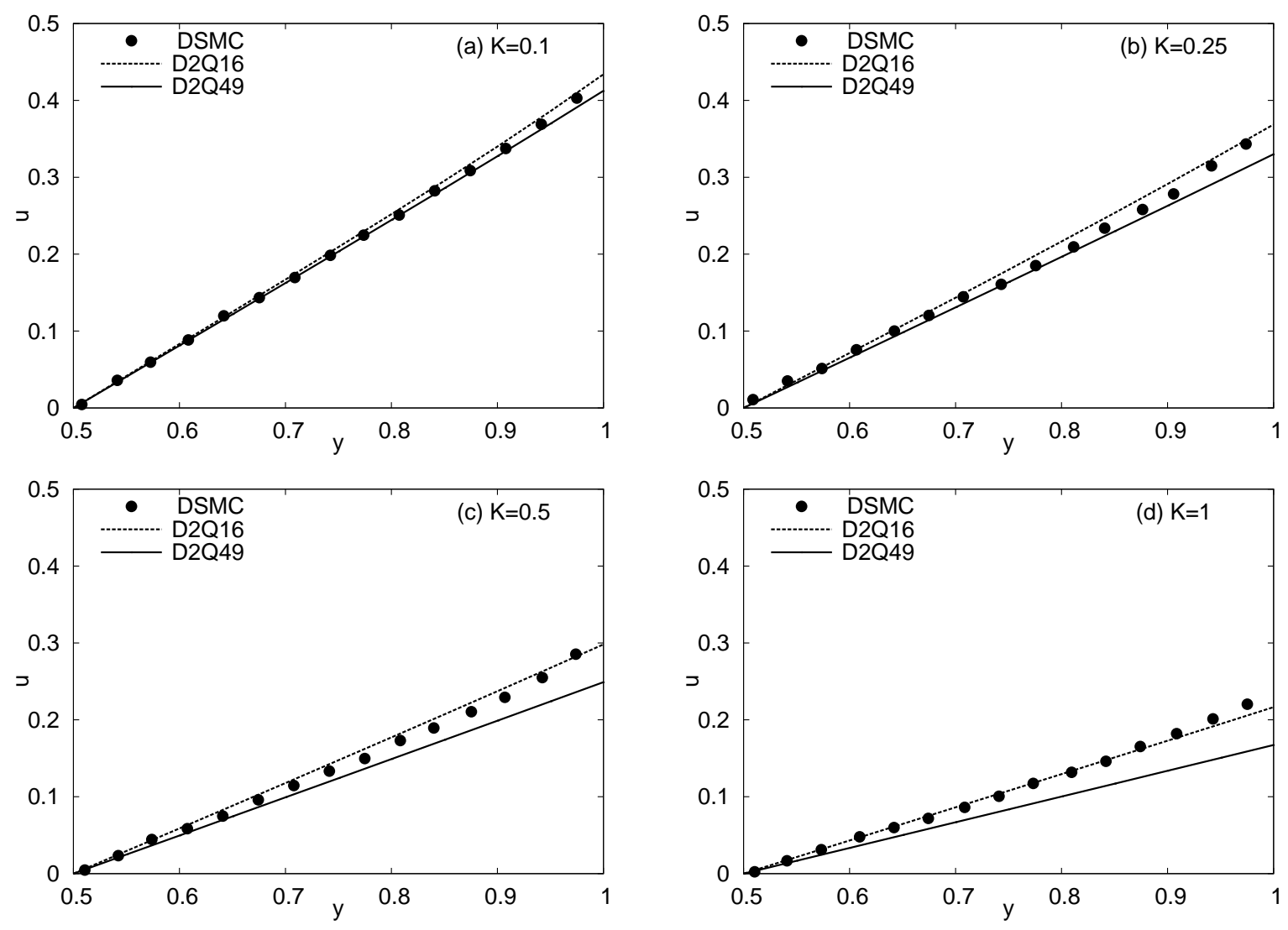

FIG. 8. Nondimensional velocity profiles for planar Couette flows where the velocity is normalized by the velocity difference between the two plates. Comparison of the LB models with the DSMC data reported in Ref.[24], where $K=\sqrt{\frac{\pi}{2}} K n$. 

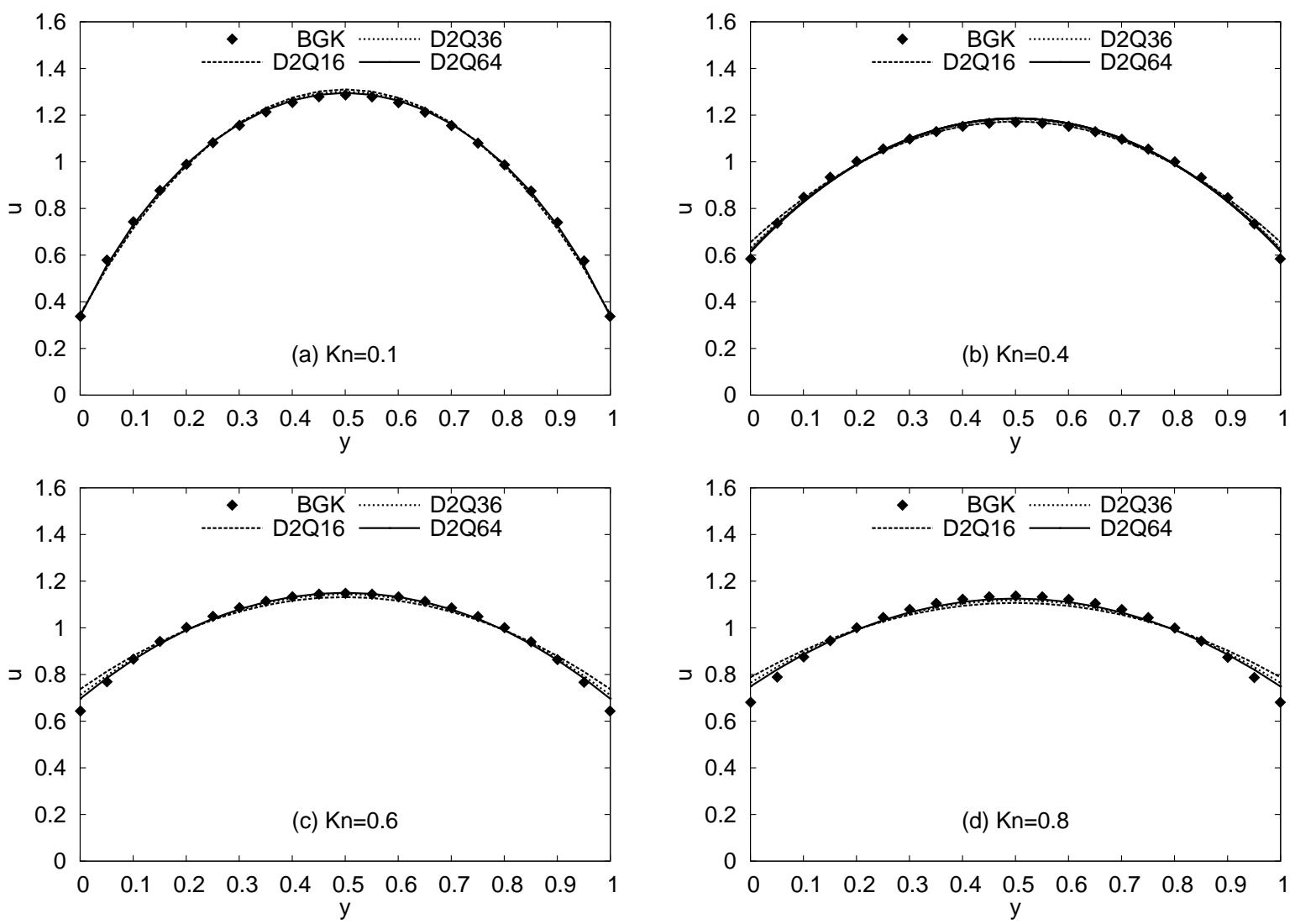

FIG. 9. Nondimensional velocity profiles for the force-driven Poiseuille flows where the velocity is normalized by the average velocity. Comparison of the even-order LB models with the linearized BGK solution.
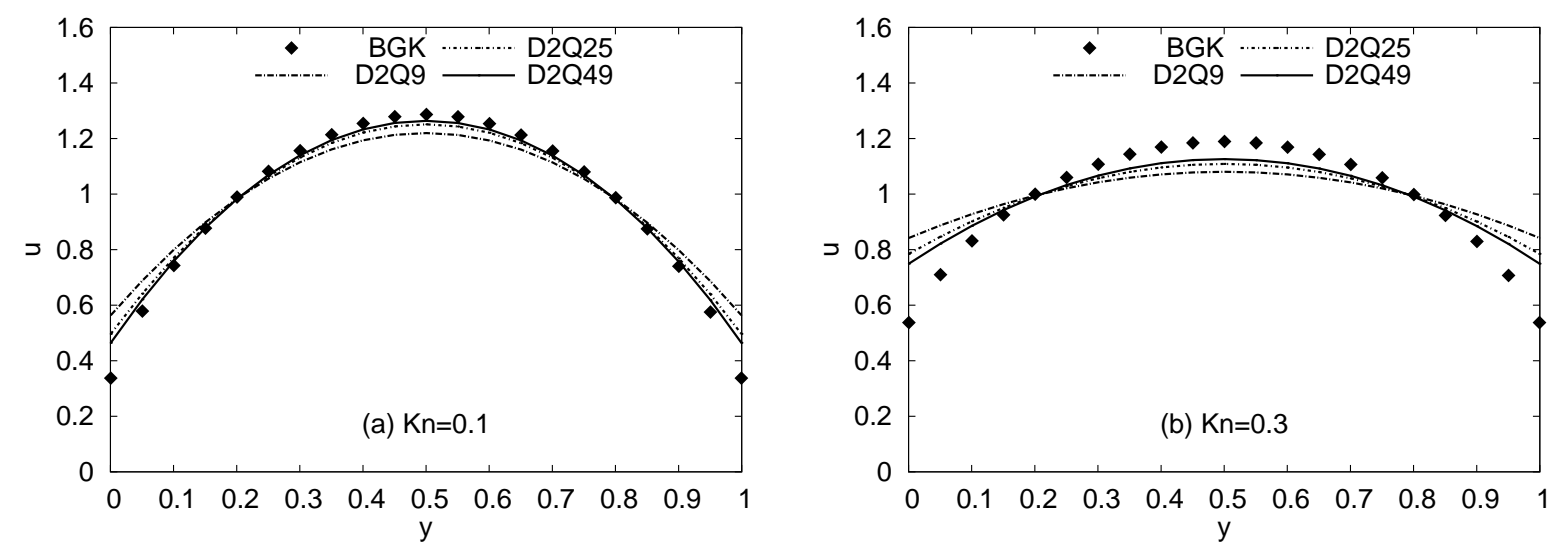

FIG. 10. Nondimensional velocity profiles for the force-driven Poiseuille flows where the velocity is normalized by the average velocity. Comparison of the odd-order LB models with the linearized BGK solution. 

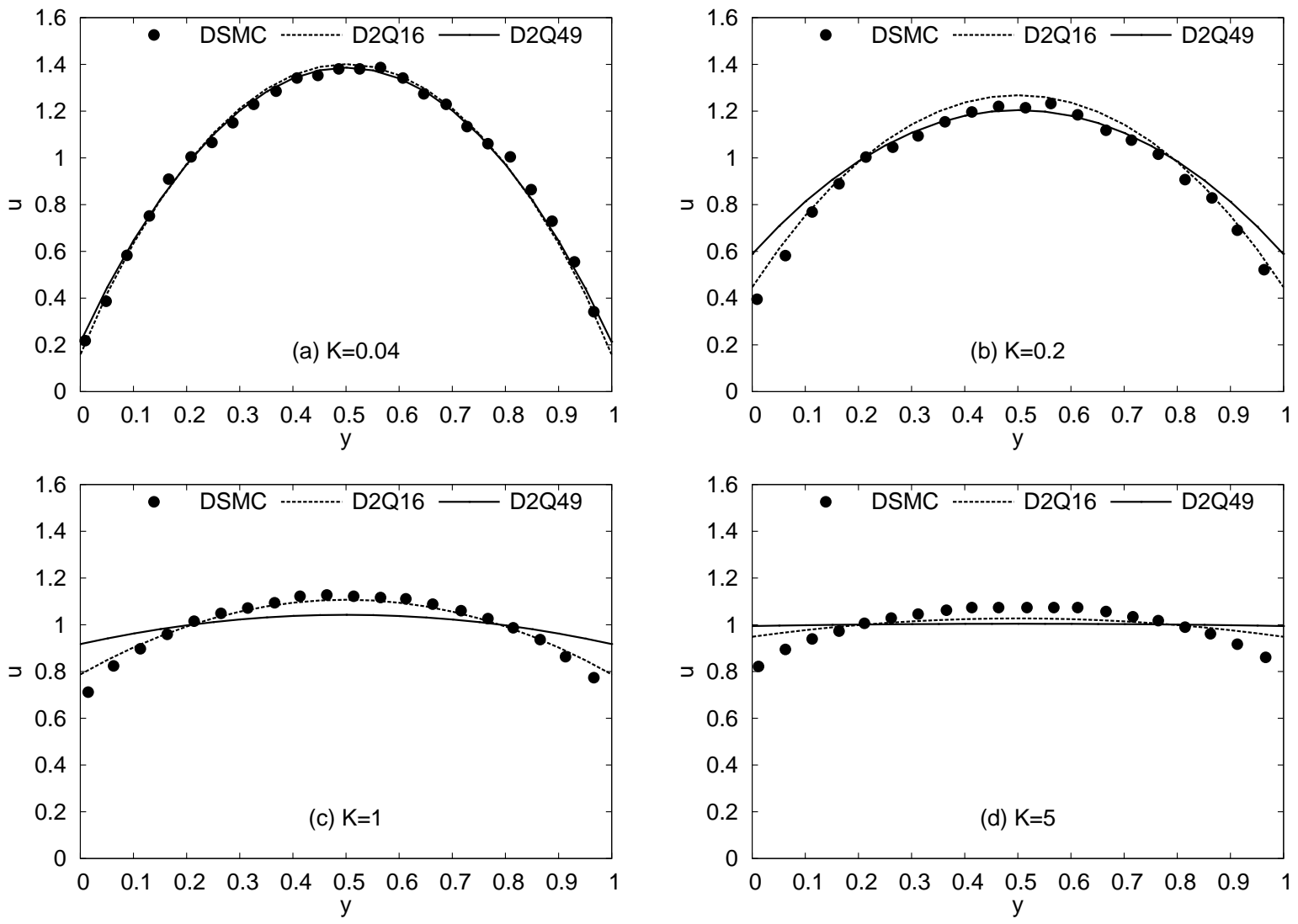

FIG. 11. Nondimensional velocity profiles for the force-driven Poiseuille flows where the velocity is normalized by the average velocity. Comparison of the LB models with the DSMC data reported in Ref.[24] where $K=\sqrt{\frac{\pi}{2}} K n$.
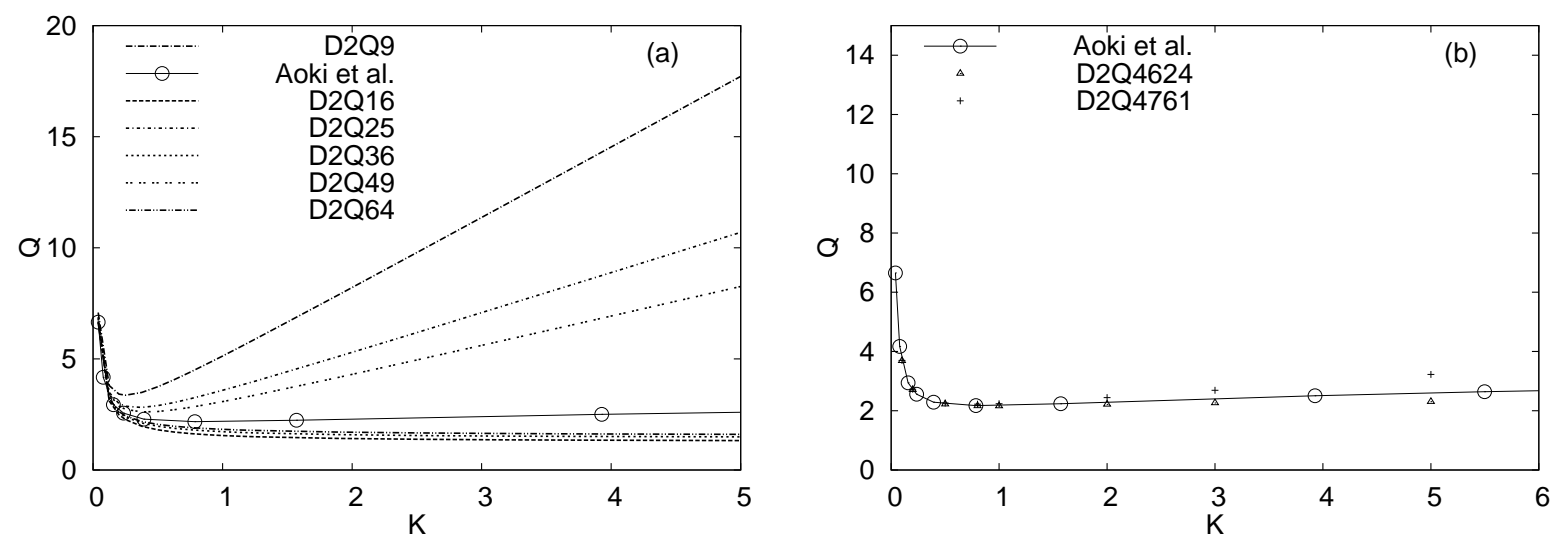

FIG. 12. The comparison study of different order LB models for the Knudsen minimum. The normalized mass flux Q is $\frac{1}{4 u_{c} l K n} \int_{0}^{l} u \mathrm{~d} y$ where $u_{c}$ is the centerline velocity of the Navier-Stokes equation with no-slip boundary condition. The circles are the data from Aoki et al.[47]. The original data are presented with the Knudsen number definition of $(\mu \sqrt{8 R T}) /(\sqrt{\pi} p l)$. They are converted to be consistent with the definition of $K=\sqrt{\frac{\pi}{2}} K n$. 